\title{
An Improved Deep Learning Based Cervical Cancer Detection Using a Median Filter Based Preprocessing
}

\author{
Zehra Karapınar Senturk $^{1 *}$, Suleyman Uzun ${ }^{2}$ \\ ${ }^{1 *}$ Duzce University, Faculty of Engineering, Department of Computer Engineering, Duzce, Turkey, (ORCID: 0000-0003-3116-1985), zehrakarapinar@duzce.edu.tr \\ ${ }^{2}$ Sakarya Applied Sciences University, Faculty of Eng, Department of Computer Eng., Sakarya, Turkey (ORCID: 0000-0001-8246-6733), suleymanuzun@subu.edu.tr
} (International Conference on Design, Research and Development- 20 - 22 October 2021)

(DOI: $10.31590 /$ ejosat.1045538)

ATIF/REFERENCE: Karapinar Senturk Z. \& Uzun, S. (2021). An Improved Deep Learning Based Cervical Cancer Detection Using a Median Filter Based Preprocessing. European Journal of Science and Technology, (32), 50-58.

\begin{abstract}
Cervical cancer is one of the prevalent type of cancer among women although its treatment success is the highest when compared to other types of cancer once diagnosed. Automatic classification of cervical cancer is essential to accelerate the treatment process and increase the survival rate of the patients. Inadequate awareness, deficiency of medical opportunities, and expensive screening procedures increase the death rates. This common cancer is frequently screened by several imaging tests including Pap smear, cervicography and colposcopy. The decisions are made by the help of these tests, but structural complexities of cervical cells may complicate the decision. Recent developments in neural networks show remarkable achievements in disease diagnosis. Also, transfer learning draws the attention of most of the researchers because of its advantages. This paper presents a transfer learning based cervical cancer detection method for early diagnosis. Pap smear images were preprocessed using median filter before training the deep learning model in order to remove noise from the images for better classification. Cancerous and non-cancerous cervical cells are distinguished through pre-trained networks. Five popular pre-trained networks which are SqueezeNet, VGG-19, AlexNet, ResNet-50 and InceptionV3 have been utilized and compared for the problem. SqueezeNet achieved the best validation accuracy $(96.90 \backslash \%)$ when compared to other neural structures and this performance makes the proposed method the best among other unsupervised approaches in the literature for cervical cancer diagnosis. Additional experiments also proved the success of the proposed model for the classification of two similar classes, namely Parabasal and Metaplastic cells. The results demonstrate that the proposed approach can provide a confidential, cheap, and fast decision support system for cervical cancer diagnosis.
\end{abstract}

Anahtar Kelimeler: Cervical cancer diagnosis, Convolutional neural networks, Transfer learning, Pap smear images

\section{Medyan Filtre Tabanlı Önişlem Kullanılarak Geliştirilmiş Bir Derin Öğrenme Temelli Rahim Ağzı Kanseri Tespiti}

$\ddot{O} z$

Rahim ağzı kanseri, teşhis edildikten sonra diğer kanser türlerine kıyasla tedavi başarısı en yüksek olanıdır ve kadınlar arasında en sık görülen kanser türlerinden biridir. Rahim ağzı kanserinin otomatik sınıflandırılması, tedavi sürecini hızlandırmak ve hastaların hayatta kalma oranlarını artırmak için çok önemlidir. Yetersiz farkındalık, tıbbi imkânların yetersizliği ve pahalı tarama prosedürleri ölüm oranlarını artırmaktadır. Bu yaygın kanser sıklıkla Pap smear, servikografi ve kolposkopi gibi çeşitli görüntüleme testleri ile taranır. Kararlar bu testler yardımıyla verilir, ancak servikal hücrelerin yapısal karmaşıklıkları kararı zorlaştırabilir. Sinir ağlarındaki son gelişmeler, hastalık teşhisinde dikkate değer başarılar göstermektedir. Ayrıca, transfer öğrenme avantajlarından dolayı çoğu araştırmacının dikkatini çekmektedir. Bu makale erken teşhis için transfer öğrenme tabanlı bir serviks kanseri tespit yöntemi sunmaktadır. Pap smear görüntüleri, daha iyi sınıflandırma için, görüntülerden gürültüyü çıkarmak amacıyla derin öğrenme modelinin eğitiminden önce medyan filtresi kullanılarak ön işleme tabi tutulmuştur. Kanserli ve kanserli olmayan servikal hücreler, önceden eğitilmiş ağlar aracılığıyla ayırt edilir. SqueezeNet, VGG-19, AlexNet, ResNet-50 ve InceptionV3 isimli beş popüler önceden eğitilmiş ağ kullanılmış ve problem için karşılaştırılmıştır. SqueezeNet diğer sinirsel yapılarla karşılaştırıldığında en iyi test doğruluğunu (\%96.90) elde etmiştir ve bu performans önerilen yöntemi serviks kanseri teşhisi için literatürdeki diğer denetimsiz yaklaşımlar arasında en iyisi yapmaktadır. Ek deneyler, Parabasal ve Metaplastik hücreler olmak üzere iki benzer sınıfın sınıflandırılması için önerilen modelin başarısını da kanıtladı. Sonuçlar, önerilen yaklaşımın rahim ağzı kanseri teşhisi için güvenilir, ucuz ve hızlı bir karar destek sistemi sağlayabileceğini göstermektedir.

Keywords: Rahim ağzı kanseri teşhisi, Evrişimli sinir ağları, Transfer öğrenme, Pap smear görüntüleri

*Corresponding Author: zehrakarapinar@duzce.edu.tr 


\section{Introduction}

As a fourth common cancer type among women, cervical cancer causes hundreds of thousands of women deaths worldwide. World Health Organization (WHO) recorded 570000 women diagnosed as cervical cancer and 311000 cervical cancer death in 2018 (World Health Organization, n.d.). Treatment success of cervical cancer is the best among other cancer types according to WHO. The most important problem for cervical cancer prevention stays as early detection. Screening and treating precancerous lesions are performed to save women from cervical cancer. But lack of medical facilities, lack of experts and expensive screening procedures expose most of the women in underdeveloped countries to tough cancer process. Computer-aided decision support systems play an important role to overcome these issues. In the recent years, computerized decision systems have been frequently used for medical purposes. Especially for cancer there are many papers published on machine learning based diagnosis in the last decade. Prostate cancer (Nitta et al., 2019), gastric cancer (Dong et al., 2020), breast cancer (Shi et al., 2009), (Senturk \& Kara, 2014), (Ekici \& Jawzal, 2020), (Guo et al., 2019), pancreatic cancer (Hashimoto et al., 2019), and lung cancer (H. Li et al., 2018) are the examples of computer-aided cancer diagnosis.

Determination of whether a person has cervical cancer has been searched in several studies. Xue et al. (Xue et al., 2010) developed a web-based segmentation method to identify certain cervical tissues using cervicography images. The system can archive, retrieve, compress, and analyze cervix images. Intensity, saturation and gradient information were used for the segmentation of cervix images. Arya et al. (Arya et al., 2016) used morphological operations for the segmentation of Pap smear images. Support Vector Machine (SVM) classifier decided whether the person has cancer or not. Fuzzy c-means clustering has been used by Indrabayu et al. (Indrabayu et al., 2017) in order to extract the nuclei region that may be the sign of cervical cell abnormality. Bhowmik et al. (Bhowmik et al., 2018) also extracted nuclei region using their own dataset. Linear SVM has been exploited by 12 shape features. Feature selection improved the classification performance to $97 \%$. 70 single cell Pap smear images of Herlev dataset has also been used in the experiments of the 7-class classification problem and 93\% precision and 94\% recall have been obtained. Yang et al. (W. Yang et al., 2019) analyzed the risk factors of cervical cancer. The data has been gathered from a hospital in Venezuela. 36 factors have been evaluated by MLP and random forest. "age" and "number of sexual partner" factors were found to be the most effective for cervical cancer. Yang et al. (Z. Yang et al., 2019) used colposcopy images for cancer detection. Colposcopy provides a magnified and illuminated view of cervix as a medical diagnosis method. Multi-spectral narrow-band imaging has been performed for cervical tissue images. K-means clustering algorithm classified cancer tissues with $89 \%$ accuracy and $84 \%$ sensitivity. Win et al. (Win et al., 2019) classified cancer cases using bagging ensemble classifier from Pap smear images. They first extracted features using Random Forests and used several machine learning algorithms. Herlev dataset was used and $97.83 \%$ and $81.54 \%$ accuracy were achieved for 2-class and 7-class classification problem.
Recent developments in neural network technology made its usage very prevalent in many applications. Deep neural networks have made remarkable success over other classification models for medical diagnosis using different source of medical images (Lundervold \& Lundervold, 2019). It is the state-of-the-art method now for wide range of fields. Recognizing natural images by computers was quite tough until convolutional neural networks were introduced. It is now one of the top 10 study area (Cearley et al., n.d.). Ke et al. (Ke, Jiang, et al., 2019) segmented nuclei region by a deep learning (DL) based approach with $87 \%$ sensitivity. Mas et al. (Martínez-Más et al., 2018) constituted their cervical cell dataset and they used DL for the classification of benign and malignant cells with $78 \%$ accuracy. DL and some extracted features were exploited to reduce noise in cancer detection process by $\mathrm{Ke}$ et al. (Ke, Deng, et al., 2019). A composite architecture including ResNet and U-Net for the classification of the subparts of cervical tissue. Less false positives have been achieved by noise removal. Mouser and Ouadfel (Mousser \& Ouadfel, 2019) used DL for feature extraction from Pap smear images and these features were used in the Multi-Layer Perceptron (MLP). They first detected the cells and then the cells were segmented. Herlev dataset was used and $89.22 \%$ accuracy obtained using ResNet50 architecture. Immunohistochemistry images, which are used for the operation of identifying proteins in cells of a tissue (Immunohistochemistry, 2020), were used by $\mathrm{Li}$ et al. (C. Li et al., 2019) for cancer diagnosis by means of transfer learning and $77.3 \%$ accuracy was obtained. Plissiti et al. extracted CNN-based features from Pap smear images and obtained 95\% accuracy.

A new cervical cell dataset has been introduced by Plissiti et al. (Plissiti et al., 2018) in 2018 and they compared the classification performance of SVM, MLP and CNN to distinguish 5 cell types. The best performance was achieved by VGG-19 adapted CNN and 95.35\% average accuracy was obtained. Haryanto et al. (Feng et al., 2019) also classified Pap smear images in SIPaKMeD dataset by $\mathrm{CNN}$ and they showed that zero padding improves the classification performance of AlexNet architecture.

In the proposed study, we used SIPaKMeD dataset proposed by Plissiti et al. (Plissiti et al., 2018) since it is the most recent cervical cell image dataset and consists of more images when compared to Herlev dataset.

Main contributions of the proposed early cervical cancer diagnosis system are given below.

- Five pre-trained neural network architectures have been compared for the classification of cervical cancer for the first time.

- A lightweight decision support system has been proposed by a deep learning based approach through less number of smaller filters for convolution.

- The highest accuracy in the literature has been achieved for cervical cancer for which the diagnosis is lifesaving.

- An easy and effective preprocessing step was added to the process. median filtered images were trained and the performance of the deep learning model was improved so.

- Two-class classification as cancer and non-cancer has been performed instead of five-class classification for cervical cancer diagnosis. 

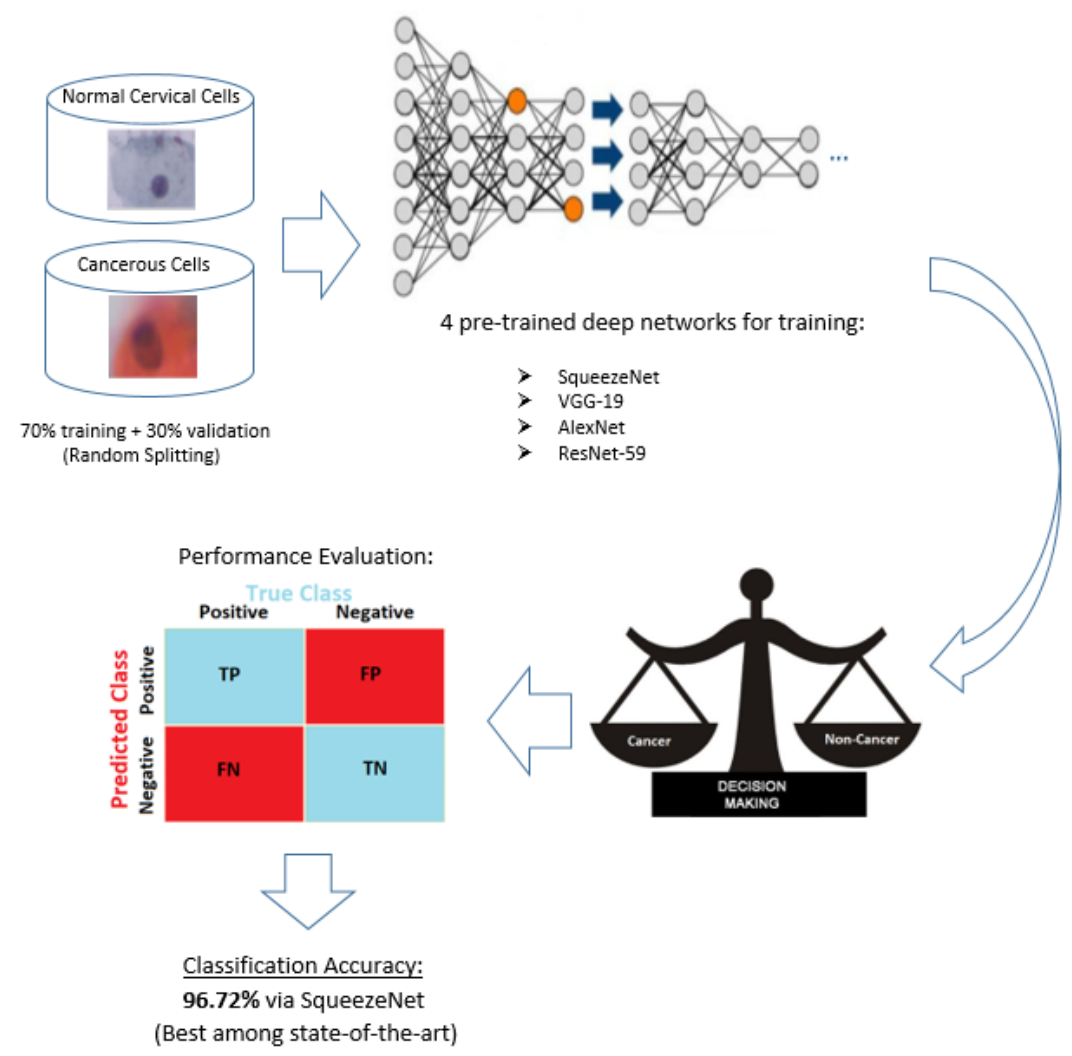

Figure 1. Work flow of the proposed transfer learning based cervical cancer detection system

The rest of the paper has been organized as follows: Section 2 gives the details of the material and the methods used in the study, Section 3 gives the experimental results of the proposed cancer detection approach and Section 4 concludes the paper.

\section{Materials and Methods}

Deep learning has been utilized in this study in order to diagnose cervical cancer from Pap smear images. Transfer learning based approach has been adapted to the problem. Different pre-trained networks have been applied and compared. SqueezeNet, VGG-19, AlexNet, ResNet-50 and InceptionV3 networks have been employed for cervical cancer detection. An illustration of the proposed transfer learning based early diagnosis system is shown in Figure 1.

\subsection{SIPaKMeD Dataset}

SIPaKMeD dataset is the most recent open cervical image dataset. Its name comes from the first letters of the cell type names in the dataset, i.e. SI for Superficial/Intermediate, P for Parabasal, $\mathrm{K}$ for Koilocytotic, M for Metaplastic, D for Dyskeratotic. It has been donated in 2018 to facilitate early detection of cervical cancer. Plissiti et al. (Plissiti et al., 2018) cropped 4049 single cell images from 966 cervical tissue images which are obtained from Pap smear slides. In this dataset, the images of five cell types were included. Figure 2 shows sample images provided in the dataset. Types of the cells are defined based on the position and the maturation level. Superficial/intermediate and parabasal cells are the normal or non-cancerous cells. Koilocytotic and dyskeratotic cells are abnormal cells and metaplastic cells are benign but suspicious precancerous lesions, i.e. they signal cancer probability. 1618 of the images are in normal class and 2449 of them are abnormal cells. In this study, we will classify cervical cells as normal or abnormal to define non-cancerous and cancerous cells in an early stage.
In this study, random $70 \%$ of the dataset was used in training and the rest $30 \%$ was used for testing the classification performance of the proposed deep model.
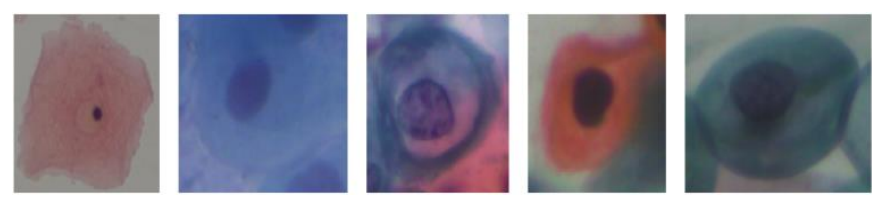

Figure 2. Superficial/intermediate, parabasal, koilocytotic, dyskeratotic, and metaplastic cell images from left to right [25]

\subsection{Transfer Learning Based Cervical Cancer Detection}

Image classification is one of the fundamental biological abilities of human visual perception system and it is a hot and important topic in computer vision field (Feng et al., 2019). Recent efforts of the researchers mostly focus on the accuracy improvement of the classification tasks especially for large datasets using advanced methods. Traditional methods show sufficient performance on small datasets, but as for large datasets, the results are not satisfactory. CNN has recently been used as a lifesaving methodology for large image datasets. CNN achieves extremely high classification accuracies in many applications (Thomas et al., 2021) - (Gautam \& Raman, 2021). This achievement comes from its learning ability thanks to a complex neural architecture. Image processing got easy with the trained CNNs for large-scale object recognition problems [34]. The information learned by $\mathrm{CNN}$ can be transferred to other datasets to solve other problems. Using the activations of CNNs pretrained on object recognition as the ad-hoc feature representation for visual information processing tasks has been frequently preferred by the computer vision community (Gatys et al., 2017). 


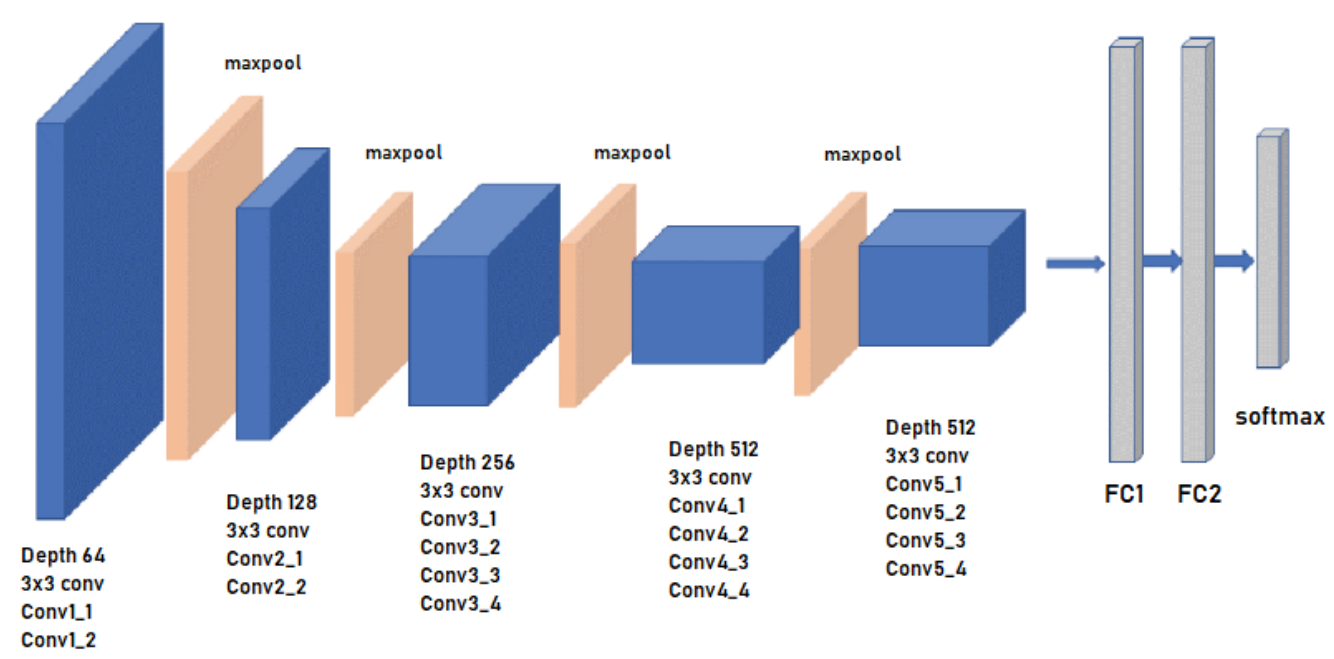

Figure 3. Architecture of VGG-19 (Arishanapally, 2019)
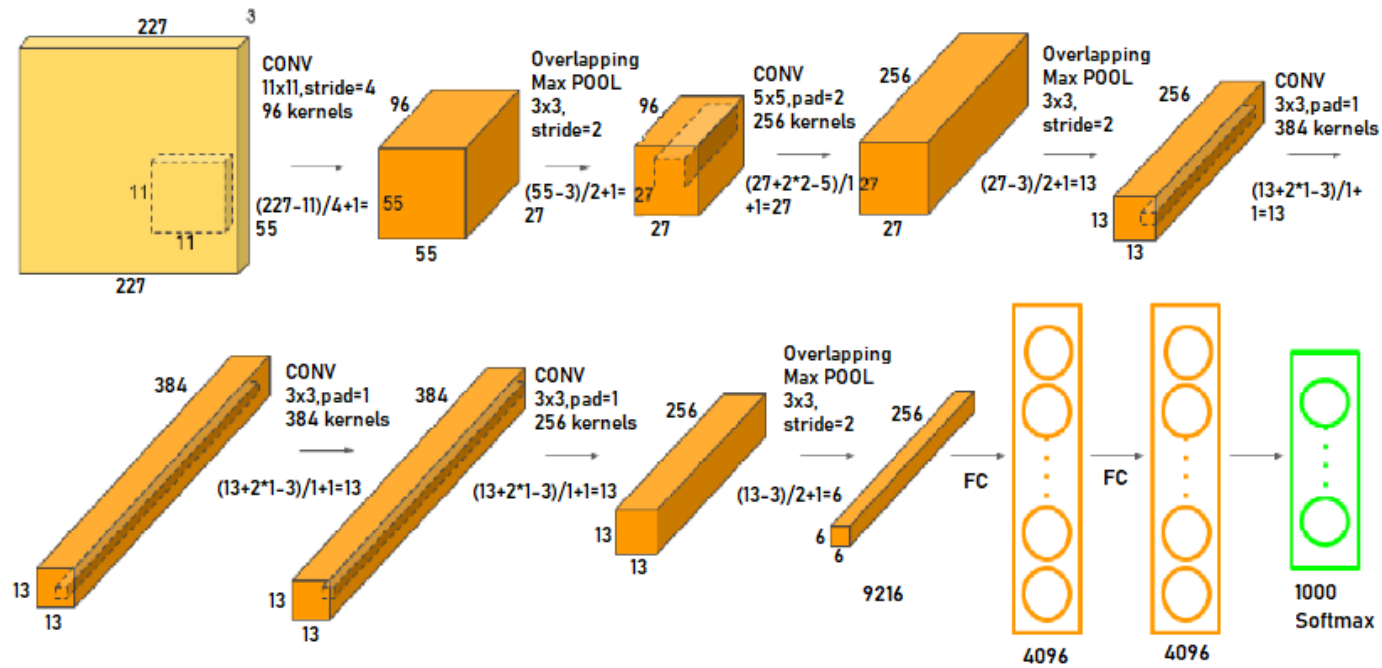

Figure 4. Architecture of AlexNet (Çevik \& Dandıl, 2019)

Gatys et al. highlighted that state-of-the-art CNNs extract perceptual image properties (Gatys et al., 2017). From this point of view, we planned to diagnose cervical cancer using pre-trained popular neural network architectures. In this study, SqueezeNet, VGG-19, AlexNet, ResNet-50 and InceptionV3 networks have been employed to distinguish cancerous and non-cancerous cells in women cervix.

VGGNet concentrates on developing deeper networks for more accurate classification. It constructs a thriving CNN with 16 to 19 layers by iterative $3 \times 3$ convolutional kernels and $2 \times 2$ maxpooling layers (Feng et al., 2019). The architecture of VGG-19 used in the experiments are shown in Figure 3.

AlexNet has 60 million parameters with eight convolutional, 3 pooling, and 3 fully connected layers (Feng et al., 2019). Its architecture is shown in Figure 4. ReLU activation function, not sigmoid, is used in its layers. It is known to be the basis of modern deep CNN.

The deeper network does not always guarantee the more accuracy since the gradient information may vanish during training and ResNet has been developed for this problem. ResNet uses a shortcut connection between residual blocks in order to use all the information in previous layers. It may train up to 152 layers by the use of residual blocks (Feng et al., 2019). SqueezeNet has been developed to fit the computational limitations that are mostly forced by the above networks. It is a lightweight architecture with less number of $3 \times 3$ filters but mostly $1 \times 1$ filters and less input channels for $3 \times 3$ filters (Feng et al., 2019). Down sampling is delayed in order not to lose information of the previous layers since information loss results with less accurate classification. It is fifty times smaller than AlexNet. Inception network architecture uses less parameters and increases the depth of the network. It has four versions. InceptionV3 was developed by Keras. It takes 299x299 color input images by default. It uses convolutional kernel splitting method in order to reduce the number of parameters for faster training process (D et al., 2020). $3 \times 1$ and $1 \times 3$ convolutions are used instead of $3 \times 3$ convolution as shown in Figure 5.

Besides evaluating the performance of these pre-trained networks for cervical cancer diagnosis using the original images of SIPaKMeD dataset, we also performed additional experiments which include a preprocessing step before training. We thought that exposing the original images to median filter may remove the noises from the images and may improve classification performance. Therefore, we additionally performed a median filter based preprocesing for the input images before training. 


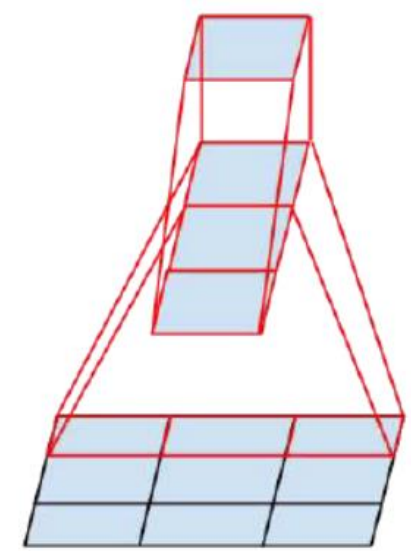

Figure 5. 3x1 and 1x3 convolutions instead of 3x3 convolution (Tsang, 2018)

\section{Results}

\subsection{Performance Metrics}

In order to evaluate the classification performance of the proposed cervical cancer diagnosis system, we used several performance metrics which are frequently used in the literature. Accuracy, precision, sensitivity, and specificity are the metrics that we measure for the problem stated in this paper. Confusion matrix has been obtained for the experiments and True Positive (TP), False Positive (FP), True Negative (TN), and False Negative $(\mathrm{FN})$ values in this matrix are used to calculate the mentioned metrics. Accuracy is the rate of correct classifications to all classifications as given in Equation 1. Precision, sensitivity, and specificity are calculated as given in Equation 2 to Equation 4 respectively.

$$
\begin{gathered}
\text { Accuracy }=\frac{T P+T N}{T P+T N+F P+F N} \\
\text { Sensitivity }=\frac{T P}{T P+F N} \\
\text { Specificity }=\frac{T N}{T N+F P} \\
\text { Precision }=\frac{T P}{T P+F P}
\end{gathered}
$$

Although accuracy is a good performance metric to show the success of the classifier, in many health based classification problems sensitivity becomes more important. Correct determination of patients is more vital than correct determination of healthy people since incorrect decision about a patient may delay his treatment time which is crucial in cancer-like diseases. Therefore, high sensitivity signals good classification performance besides accuracy.

\subsection{Experimental Results}

In this paper, we considered the problem in hand as a 2-class classification problem and compared the performance of five pretrained neural networks on classifying cervical cells. In order to remove noise from the input images, we applied median filter to all the images that we used in training and testing. The size of training dataset in this study was relatively small and a deep neural network needs larger datasets. Therefore, we used data augmentation technique in this study and added three more cell images for every image in the dataset. The newly added images were obtained by shift and rotate operations in vertical and horizontal directions. In this way, the dataset that we used in our experiments was quadrupled.

The experiments of the proposed cervical cancer detection system have been conducted using MATLAB's Transfer Learning Toolbox. $70 \%$ of the samples was used in the training of the model and 30 of them was used for validation. Images are of size $227 \times 227 \times 3$. Validation images were not processed by the network during training. The network did not see these validation images. Learning rate was 0.0001 and GPU environment was used. The number of layers in SqueezeNet, VGG-19, AlexNet, ResNet-50 and InceptionV3 networks are 68, 47, 25, 177, and 315 respectively.

The experiments were performed in three staages. In the first stage, the original images of SIPaKMeD dataset were given to the previously stated deep models. That is, no preprocessing was performed. The training parameters were as follows: initial learning rate $=0.0001$, learning rate drop factor $=0.1$, L2Regularization $=0.0001$, momentum $=0.9$. The confusion matrix with this parameter adjustments is given in Table 1 and the performance metrics obtained for each pre-trained network for the corresponding confusion matrix is shown in Table 2. The best accuracy belongs to the classification using SqueezeNet for cervical cell classification.

Table 1. Confusion matrix for the first experiment

\begin{tabular}{|l|c|c|c|c|}
\hline Network & TP & TN & FP & FN \\
\hline SqueezeNet & 726 & 192 & 3 & 57 \\
\hline VGG-19 & 646 & 247 & 83 & 2 \\
\hline AlexNet & 723 & 190 & 6 & 59 \\
\hline Resnet-50 & 728 & 165 & 1 & 84 \\
\hline InceptionV3 & 625 & 233 & 104 & 16 \\
\hline
\end{tabular}

Table 2. Performance metrics for the first experiment

\begin{tabular}{|l|c|c|c|c|}
\hline Network & Accuracy & Precision & Sensitivity & Specificity \\
\hline SqueezeNet & $\mathbf{0 . 9 3 9}$ & 0.996 & 0.927 & 0.985 \\
\hline VGG-19 & 0.913 & 0.886 & $\mathbf{0 . 9 9 7}$ & 0.748 \\
\hline AlexNet & 0.934 & 0.992 & 0.925 & 0.969 \\
\hline Resnet-50 & 0.913 & $\mathbf{0 . 9 9 9}$ & 0.897 & $\mathbf{0 . 9 9 4}$ \\
\hline InceptionV3 & 0.877 & 0.957 & 0.975 & 0.691 \\
\hline
\end{tabular}

The second stage was also with no preprocessing, but we wanted to show the effect of L2Regularization parameter. Regularization parameter is used to reduce overfitting. Setting regularization parameter to zero may reduce the generalizing capability of the network. Its value is mostly on a logarithmic scale between 0 and 0.1 . It adds some penalities to the weights. In this phase of the experiments, we changed L2Regularization parameter as 0.001 while it was 0.0001 in the previous experiment. Other parameters were preserved. As a result of this parameter adjustment, the performances of all network models were improved. The confusion matrix is given in Table 3 and the performance metrics obtained for each pre-trained network for the corresponding confusion matrix is shown in Table 4 . The best accuracy again belongs to the classification using SqueezeNet for cervical cell classification. 
Table 3. Confusion matrix for the second experiment

\begin{tabular}{|l|c|c|c|c|}
\hline Network & TP & TN & FP & FN \\
\hline SqueezeNet & 728 & 204 & 1 & 45 \\
\hline VGG-19 & 698 & 224 & 31 & 25 \\
\hline AlexNet & 711 & 208 & 18 & 41 \\
\hline Resnet-50 & 691 & 238 & 38 & 11 \\
\hline InceptionV3 & 728 & 135 & 1 & 114 \\
\hline
\end{tabular}

Table 4. Performance metrics for the second experiment

\begin{tabular}{|l|c|c|c|c|}
\hline Network & Accuracy & Precision & Sensitivity & Specificity \\
\hline SqueezeNet & $\mathbf{0 . 9 5 3}$ & $\mathbf{0 . 9 9 9}$ & 0.942 & $\mathbf{0 . 9 9 5}$ \\
\hline VGG-19 & 0.943 & 0.957 & 0.965 & 0.878 \\
\hline AlexNet & 0.940 & 0.975 & 0.945 & 0.920 \\
\hline Resnet-50 & 0.950 & 0.948 & $\mathbf{0 . 9 8 4}$ & 0.862 \\
\hline $\begin{array}{l}\text { InceptionV } \\
3\end{array}$ & 0.882 & $\mathbf{0 . 9 9 9}$ & 0.865 & 0.993 \\
\hline
\end{tabular}

Last but not least, the third phase include data preprocessing step before training starts. Images in SIPaKMeD dataset were filtered using median filter in order to reduce noise and improve the classification performance. Median filter is the best known and most widely used order statistics based filter and usually performs well in the existance of additive white Gaussian and long-tailed additive noise (Pitas \& Venetsanopoulos, 1990). It is widely used in many applications since 1970 because of its simplicity and good performance. We also used median filter for the classification of cervical cell images and evaluated its performance. The confusion matrix of the experiment with median filter based preprocessing is given in Table 5 and the performance metrics obtained for each pre-trained network for the corresponding confusion matrix is shown in Table 6 . The best accuracy again belongs to the classification using SqueezeNet for cervical cell classification and the performance of the model with median based preprocessing is the best among other models.

Table 5. Confusion matrix for the third experiment

\begin{tabular}{|l|c|c|c|c|}
\hline Network & TP & TN & FP & FN \\
\hline SqueezeNet & 707 & 241 & 22 & 8 \\
\hline VGG-19 & 726 & 203 & 3 & 46 \\
\hline AlexNet & 715 & 199 & 4 & 50 \\
\hline Resnet-50 & 713 & 221 & 16 & 28 \\
\hline InceptionV3 & 692 & 208 & 37 & 41 \\
\hline
\end{tabular}

Table 6. Performance metrics for the third experiment

\begin{tabular}{|l|c|c|c|c|}
\hline Network & Accuracy & Precision & Sensitivity & Specificity \\
\hline SqueezeNet & $\mathbf{0 . 9 6 9}$ & 0.970 & $\mathbf{0 . 9 8 9}$ & 0.916 \\
\hline VGG-19 & 0.950 & $\mathbf{0 . 9 9 6}$ & 0.940 & $\mathbf{0 . 9 8 5}$ \\
\hline AlexNet & 0.945 & 0.995 & 0.935 & 0.980 \\
\hline Resnet-50 & 0.955 & 0.978 & 0.962 & 0.932 \\
\hline InceptionV3 & 0.920 & 0.949 & 0.944 & 0.849 \\
\hline
\end{tabular}

\section{Discussion}

The accuracy values obtained by SqueezeNet based deep learning approach with median filter based preprocessing for the early diagnosis of cervical cancer are remarkable for SIPaKMeD cell dataset. SqueezeNet gave the best true decision numbers with 948 correct decisions over 978 cases. It can be deduced that the proposed method is acceptable for the detection of cervical cancer based on histopathological images when the accuracy rates are considered. Its other metrics also show the success of the proposed method. This performance proves that the proposed method can be reliably and efficiently used for the discrimination of cancer (or pre-cancer) cases from the non-cancerous cases. The method can be used as an assistant to the doctors for an efficient diagnosis for cervical cancer patients. Besides, SqueezeNet provides a lightweight solution to cervical cancer detection problem. Therefore, a fast and cheap decision support system has been provided in the study in order to ensure high expert standards for the women with few economical and medical facilities in underdeveloped countries.

The main problem in the test phase was that training reaches the threshold values very fast and so not all of the steps were completed. The reason is that pre-trained networks do not reach to the defined criteria and the error cannot be reduced no more. For less amount of error, the number of images can be increased.

Although there are several papers in the literature for cervical cancer diagnosis, they use Herlev dataset instead of SIPaKMeD. (Singh \& Goyal, 2020), (Dongyao Jia et al., 2020), and (Zhang et al., 2017) achieved high classification performances on Herlev dataset. However, their methods were not fully unsupervised or the methods are computationally heavy. Herlev dataset contains 917 cell images, 675 abnormal and 242 normal cells while the more recent SIPaKMeD dataset has 1618 normal and 2449 abnormal cell images. In this paper, we proposed a fully unsupervised solution to cervical cancer diagnosis problem with SIPaKMeD dataset. Therefore, we compared our results with the recent study that uses SIPaKMeD dataset.

Table 7. Comparison with the state-of-the-art for SIPaKMeD dataset

\begin{tabular}{|l|c|c|c|}
\hline Reference No & (Plissiti et al., 2018) & (Haryanto et al., 2020) & Proposed \\
\hline Method & VGG19-based & AlexNet-based & SqueezeNet-based \\
\hline Preprocessing & - & Zero padding & $\begin{array}{c}\text { Median filter based } \\
\text { preprocessing }\end{array}$ \\
\hline No of Conv. Stacks & 5 & 5 & 5 \\
\hline No of layers & 35 & 25 & 25 \\
\hline Conv. - Max pool. filter size & $3 \times 3-2 \times 2$ & $11 \times 11-5 \times 5-3 \times 3$ & $1 \times 1$ \\
\hline Depth of conv. layers in each stack & $64,128,256,512,512$ & $96,256,384,256$ & $96,128,256,384,512$ \\
\hline Fully connected layer size & $4096,4096,5$ & $4096,4096,1000$ & - \\
\hline Accuracy $(\%)$ & 95.35 & $\mathbf{8 7 . 3 2}$ & $\mathbf{9 6 . 9 0}$ \\
\hline
\end{tabular}

Table 7 compares the accuracies of the proposed method and the recent studies which uses SIPaKMeD dataset. Our method outperforms the present approaches in the literature with a lightweight training process. Accurate results have been obtained using considerably less number of parameters thanks to the design strategy of SqueezeNet. See (Win et al., 2020) for the details of 
this network. There is no fully connected layer in this framework. Instead, there are fire layers between layer 2 and layer 9. Squeeze (with $1 \times 1$ filter) and expand (with $31 \times 1$ and $33 \times 3$ filters, totally 6 filters) layers exist in this network different from the other structures. Table 3 shows that the proposed approach achieves the best accuracy with less layers and smaller filters in layers, which proves the computational convenience of the method. Win et al. (Win et al., 2020) also achieved high (98.27\%) classificaton accuracy using SIPaKMeD dataset, but they used a supervised approach for the purpose. An ensemble of linear discriminant, knearest neighbor, boosted tree, bagged tree, and support vector machines determined the classes of cervical cells either cancerous or non-cancerous. They performed many pre-processing including segmentation, feature extraction, and feature selection. Although their accuracy is better than ours, their method has drawbacks for being an unsupervised approach and requiring heavy processing. We proposed a lightweight training process with an acceptable accuracy and this enables realtime decision making.

Besides the previously considered analysis, the ability of the proposed model to distinguish parabasal cells from metaplastic ones was also tested. Plissity et al. (Plissiti et al., 2018) stated that parabasal cells have similar morphological characteristic with the metaplastic cells and it is difficult to be distinguished from them. Parabasal cells are in the non-cancerous (normal) group and metaplastic cells are accepted in the pre-cancerous (or abnormal) group. Therefore, we performed an additional test to measure the performance of the model against these two similar classes. We trained the models using only parabasal and metaplastic images and tested the performance of the pre-trained networks to distinguish these similar cell classes. 793 metaplastic and 787 parabasal images were used in the training and testing. The similar experimental steps were followed also for this problem. We first trained the network with no pre-processing. Then, we trained by adjusting L2Regularization parameter and lastly, we tested the performance after median filter based pre-processing. The best results were obtained again by including median filter based preprocessing. SqueezeNet again produced the best performance with $94.93 \%$ accuracy. It achieved very high classification accuracy even with less number of input images, which is a critical issue in deep learning applications. Deep learning models need high amount of input images in order to produce acceptable results, but in our case we achieved remarkable performance with less input images thanks to median filter based pre-processing and SqueezeNet. The corresponding confusion matrix of the best model to distinguish parabasal cells from metaplastic cells is shown in Table 8 and the performance metrics are given in Table 9.

Table 8. Confusion matrix for the classification of parabasal and metaplastic cells

\begin{tabular}{|l|c|c|c|c|}
\hline Network & TP & TN & FP & FN \\
\hline SqueezeNet & 233 & 217 & 3 & 21 \\
\hline VGG-19 & 224 & 192 & 12 & 46 \\
\hline AlexNet & 207 & 225 & 29 & 13 \\
\hline Resnet-50 & 230 & 219 & 6 & 19 \\
\hline InceptionV3 & 215 & 212 & 21 & 26 \\
\hline
\end{tabular}

Table 9. Performance metrics for the classification of

parabasal and metaplastic cells

\begin{tabular}{|l|c|c|c|c|}
\hline Network & Accuracy & Precision & Sensitivity & Specificity \\
\hline SqueezeNet & $\mathbf{9 4 . 9 3}$ & $\mathbf{9 8 . 7 2}$ & 91.73 & $\mathbf{9 8 . 6 3}$ \\
\hline VGG-19 & 87.76 & 94.91 & 82.96 & 94.11 \\
\hline
\end{tabular}

\begin{tabular}{|l|l|l|l|l|}
\hline AlexNet & 91.13 & 87.71 & $\mathbf{9 4 . 0 9}$ & 88.58 \\
\hline Resnet-50 & 94.72 & 97.45 & 92.36 & 97.33 \\
\hline InceptionV3 & 90.08 & 91.10 & 89.21 & 90.98 \\
\hline
\end{tabular}

SqueezeNet network misclassified only 3 metaplastic cells as parabasal and 21 parabasal cells as metaplastic. 450 test samples over 474 were classified correctly. This achievement proves that SqueezeNet can be reliably used also for the classification of morphologically similar cells, metaplastic cells and parabasal cells, after median filter based preprocesing.

\section{Conclusion}

Cervical cancer is the fourth common cancer type among women and it causes hundreds of thousands of deaths every year. The most important property of cervical cancer is that it is the most treatable cancer when compared to other cancer types. Therefore, its early detection is crucial. A deep learning based cervical cancer detection method has been presented in this paper. Pap smear images provided by SIPaKMeD dataset, a publicly available dataset, were used. The advantages of transfer learning were utilized and different pre-trained networks were compared. In addition, median filter based preprocessing was also shown to be effective for the performance improval of the deep learning model. Cancerous and non-cancerous cervical cells have been determined by the virtue of pre-trained CNNs. This study showed that SqueezeNet can be reliably used for cervical cancer diagnosis in a lightweight manner. It can be deduced that the proposed method is acceptable for the detection of cervical cancer based on histopathological images when its performance metrics are considered. It is also capable of distinguishing two similar classes, parabasal cells from metaplastic cells, properly. The method can be used as an assistant to the doctors for an efficient diagnosis for cervical cancer patients. Thus, the global burden of cervical cancer can be minimized. Besides, the proposed system can avail to understand the progress of the disease by the improvement works to be realized. Also, we will investigate other network structures for cervical cancer diagnosis.

\section{References}

Arishanapally, S. C. (2019). Building VGG19 with Keras. Medium. https://medium.com/@saicharanars/buildingvgg19-with-keras-f516101c24cf

Arya, M., Mittal, N., \& Singh, G. (2016). Cervical cancer detection using segmentation on pap smear images. $A C M$ International Conference Proceeding Series, 25-26-Augu, 15. https://doi.org/10.1145/2980258.2980311

Bhowmik, M. K., Roy, S. D., Nath, N., \& Datta, A. (2018). Nucleus region segmentation towards cervical cancer screening using AGMC-TU Pap-smear dataset. ACM International Conference Proceeding Series, 44-53. https://doi.org/10.1145/3243250.3243258

Cearley, D. W., Burke, B., Searle, S., \& Walker, M. J. (n.d.). Top 10 Strategic Technology Trends for 2018. In brilliantdude.com. Retrieved May 6, 2020, from http://brilliantdude.com/solves/content/GartnerTrends2018.p df

Çevik, K. K., \& Dandıl, E. (2019). Classification of Lung Nodules Using Convolutional Neural Networks on CT Images. 2nd International Turkish World Engineering and Science Congress, 27-35.

D, N. D. P., Zhao, L., D, C. H. W. P., \& Chang, J. F. (2020). Inception $\mathrm{v} 3$ based cervical cell classification combined with 
artificially extracted features. Applied Soft Computing Journal, 93, 1-8. https://doi.org/10.1016/j.asoc.2020.106311

Dong, D., Fang, M.-J., Tang, L., Shan, X.-H., Gao, J.-B., Giganti, F., Wang, R.-P., Chen, X., Wang, X.-X., Palumbo, D., Fu, J., Li, W.-C., Li, J., Zhong, L.-Z., De Cobelli, F., Ji, J.-F., Liu, Z.-Y., \& Tian, J. (2020). Deep learning radiomic nomogram can predict the number of lymph node metastasis in locally advanced gastric cancer: an international multi-center study. Annals of Oncology. https://doi.org/10.1016/j.annonc.2020.04.003

Dongyao Jia, A., Zhengyi Li, B., \& Chuanwang Zhang, C. (2020). Detection of cervical cancer cells based on strong feature CNN-SVM network. Neurocomputing, 411, 112-127. https://doi.org/10.1016/j.neucom.2020.06.006

Ekici, S., \& Jawzal, H. (2020). Breast cancer diagnosis using thermography and convolutional neural networks. Medical Hypotheses, 137, 109542. https://doi.org/10.1016/j.mehy.2019.109542

Feng, X., Jiang, Y., Yang, X., Du, M., \& Li, X. (2019). Computer vision algorithms and hardware implementations: A survey . Integration, the VLSI Journal, 69, 309-320. https://reader.elsevier.com/reader/sd/pii/S016792601930176 2? token=53D2C5EBAAF7604A55486ED6D40948D4F9C7 8179B7E62A07E9B73DC972CA30E58A32D661873FA500 AAEDA26F64AA61B7

Gatys, L. A., Ecker, A. S., \& Bethge, M. (2017). Texture and art with deep neural networks. Current Opinion in Neurobiology, 46, 178-186. https://doi.org/10.1016/j.conb.2017.08.019

Gautam, A., \& Raman, B. (2021). Towards effective classification of brain hemorrhagic and ischemic stroke using CNN. Biomedical Signal Processing and Control, 63, 102178. https://doi.org/10.1016/j.bspc.2020.102178

Guo, Y., Shang, X., \& Li, Z. (2019). Identification of cancer subtypes by integrating multiple types of transcriptomics data with deep learning in breast cancer. Neurocomputing, 324, 20-30. https://doi.org/10.1016/j.neucom.2018.03.072

Haryanto, T., Sitanggang, I. S., Agmalaro, M. A., \& Rulaningtyas, R. (2020). The Utilization of Padding Scheme on Convolutional Neural Network for Cervical Cell Images Classification. International Conference on Computer Engineering, Network, and Intelligent Multimedia (CENIM 2020),

$34-38$. https://ieeexplore.ieee.org/stamp/stamp.jsp?tp=\&arnumber= 9297895

Hashimoto, Y., Watanabe, Y., Takano, H., Setsuda, A., Ohno, I., Imaoka, H., Sasaki, M., Watanabe, K., Umemoto, K., Kimura, G., Shibuki, T., Kan, M., Mitsunaga, S., \& Ikeda, M. (2019). 590 - High Diagnostic Yield Using Advanced Artificial Intelligence in Cytology of Pancreatic Cancer by Eus-Fna. Gastroenterology, 156(6), S-115. https://doi.org/10.1016/s0016-5085(19)37081-7

Immunohistochemistry. (2020). Wikipedia.

Indrabayu, Fatmasari, A. R., \& Nurtanio, I. (2017). A colour space based detection for cervical cancer using fuzzy C-means clustering. ACM International Conference Proceeding Series, 137-141. https://doi.org/10.1145/3121138.3121196

Ke, J., Deng, J., \& Lu, Y. (2019). Noise Reduction with Image Inpainting: An Application in Clinical Data Diagnosis. SIGGRAPH'19.

Ke, J., Jiang, Z., Liu, C., Bednarz, T., Sowmya, A., \& Liang, X. (2019). Selective Detection and Segmentation of Cervical Cells. ICBBT'19. https://doi.org/https://doi.org/10.1145/3340074.3340081
Li, C., Xue, D., Zhou, X., Zhang, J., Zhang, H., Yao, Y., Kong, F., Zhang, L., \& Sun, H. (2019). Transfer Learning Based Classification of Cervical Cancer Immunohistochemistry Images. ISICDM'19, 24-26. https://doi.org/https://doi.org/10.1145/3364836.3364857

Li, H., Galperin-Aizenberg, M., Pryma, D., Simone, C. B., \& Fan, Y. (2018). Unsupervised machine learning of radiomic features for predicting treatment response and overall survival of early stage non-small cell lung cancer patients treated with stereotactic body radiation therapy. Radiotherapy and Oncology, 129(2), 218-226. https://doi.org/10.1016/j.radonc.2018.06.025

Lundervold, A. S., \& Lundervold, A. (2019). An overview of deep learning in medical imaging focusing on MRI. Zeitschrift Für Medizinische Physik, 29(2), 102-127. https://doi.org/10.1016/J.ZEMEDI.2018.11.002

Martínez-Más, J., Bueno-Crespo, A., Imbernón, B., Cecilia, J. M., Martínez-España, R., Remezal-Solano, M., SánchezEspinosa, A., Ortiz-Reina, S., \& Martínez-Cendán, J. P. (2018). Deep learning approach for classifying papanicolau cervical smears. ACM International Conference Proceeding Series. https://doi.org/10.1145/3229710.3229732

Mousser, W., \& Ouadfel, S. (2019). Deep Feature Extraction for Pap-Smear Image Classification: A Comparative Study. ICCTA 2019. https://doi.org/https://doi.org/10.1145/3323933.3324060

Nitta, S., Tsutsumi, M., Sakka, S., Endo, T., Hashimoto, K., Hasegawa, M., Hayashi, T., Kawai, K., \& Nishiyama, H. (2019). Machine learning methods can more efficiently predict prostate cancer compared with prostate-specific antigen density and prostate-specific antigen velocity. Prostate International, 7(3), 114-118. https://doi.org/10.1016/j.prnil.2019.01.001

Pitas, I., \& Venetsanopoulos, A. N. (1990). Median Filters. In Nonlinear Digital Filters. Springer US. https://doi.org/10.1007/978-1-4757-6017-0

Plissiti, M. E., Dimitrakopoulos, P., Sfikas, G., Nikou, C., Krikoni, O., \& Charchanti, A. (2018). SIPAKMED : A NEW DATASET FOR FEATURE AND IMAGE BASED CLASSIFICATION OF NORMAL AND PATHOLOGICAL CERVICAL CELLS IN PAP SMEAR IMAGES Dept . of Computer Science \& Engineering, University of Ioannina, Greece Dept . of Anatomy-Histology and Embryology, Facul. 25th IEEE International Conference on Image Processing (ICIP), 3144-3148. https://doi.org/10.1109/ICIP.2018.8451588

Senturk, Z. K., \& Kara, R. (2014). Breast Cancer Diagnosis Via Data Mining: Performance Analysis of Seven Different Algorithms. Computer Science \& Engineering: An International Journal, 4(1), 35-46. https://doi.org/10.5121/cseij.2014.4104

Shi, Y., Dai, D., Liu, C., \& Yan, H. (2009). Sparse discriminant analysis for breast cancer biomarker identification and classification. Progress in Natural Science, 19(11), 16351641. https://doi.org/10.1016/j.pnsc.2009.04.013

Singh, S. K., \& Goyal, A. (2020). Three stage cervical cancer classifier based on hybrid ensemble learning with modified binary PSO using pretrained neural networks. The Imaging Science Journal, 68(1), 41-55. https://doi.org/10.1080/13682199.2020.1734306

Thomas, S. M., Lefevre, J. G., Baxter, G., \& Hamilton, N. A. (2021). Interpretable deep learning systems for multi-class segmentation and classification of non-melanoma skin 
cancer. Medical Image Analysis, 68, 101915. https://doi.org/10.1016/j.media.2020.101915

Tsang, S.-H. (2018). Inception-v3 - 1st Runner Up (Image Classification) in ILSVRC 2015. Medium.

Win, K. P., Kitjaidure, Y., Hamamoto, K., \& Myo Aung, T. (2020). Computer-Assisted Screening for Cervical Cancer Using Digital Image Processing of Pap Smear Images. Applied Sciences, 10(5), 1800. https://doi.org/10.3390/app10051800

Win, K. P., Kitjaidure, Y., Phyu, M., \& Hamamoto, K. (2019). Cervical Cancer Detection and Classification from Pap Smear Images. ICBSP '19, 47-54. https://doi.org/https://doi.org/10.1145/3366174.3366178

World Health Organization. (n.d.). Cancer. Retrieved April 21, 2020, from https://www.who.int/healthtopics/cancer\#tab=tab_1

Xue, Z., Antani, S., Long, L. R., \& Thoma, G. R. (2010). An online segmentation tool for cervicographic image analysis. IHI'10 - Proceedings of the 1st ACM International Health Informatics Symposium, 425-429. https://doi.org/10.1145/1882992.1883056

Yang, W., Gou, X., Xu, T., Yi, X., \& Jiang, M. (2019). Cervical Cancer Risk Prediction Model and Analysis of Risk Factors based on Machine Learning. ICBBT'19.

Yang, Z., Yi, D., \& Shen, J. (2019). Computer-aided cervical cancer screening method based on multi-spectral narrowband imaging. ICBIP '19, 62-66. https://doi.org/10.1145/3354031.3354037

Zhang, L., Lu, L., Nogues, I., Summers, R. M., Liu, S., \& Yao, J. (2017). DeepPap: Deep convolutional networks for cervical cell classification. IEEE Journal of Biomedical and Health Informatics, 21(6), 1633-1643. https://doi.org/10.1109/JBHI.2017.2705583 